\section{Synthèse et étude structurale de $\mathrm{Na} 9 \mathrm{Cr}\left(\mathrm{MoO}_{4}\right)_{6}$}

\author{
Wassim Dridi, Ines Ennajeh* et Mohamed Faouzi Zid
}

Laboratoire de Matériaux et Cristallochimie, Faculté des Sciences de Tunis, Université de Tunis El Manar, 2092 El Manar Tunis, Tunisia. *Correspondence e-mail: inesnajeh@gmail.com

The title compound, nonasodium chromium(III) hexakis[molybdate(VI)], $\mathrm{Na}_{9} \mathrm{Cr}\left(\mathrm{MoO}_{4}\right)_{6}$ was prepared by solid-state reactions. The basic structure units are isolated polyhedral clusters composed of a central $\mathrm{CrO}_{6}$ octahedron sharing vertices with six $\mathrm{MoO}_{4}$ tetrahedra to form an open framework in which the $\mathrm{Na}^{+}$ cations are bound to the free vertices of the $\mathrm{MoO}_{4}$ tetrahedra. The $\mathrm{Cr}^{3+}$ cation has site symmetry of $32(6 a)$, one $\mathrm{Na}$ atom sits on a twofold axis (18e), and all other atoms are at general positions. The bond-valance-sum model confirms the expected values of ion charges. The title compound is isotypic with $\mathrm{Na}_{9} \mathrm{Sc}\left(\mathrm{MoO}_{4}\right)_{6}$ and $\mathrm{Na}_{9} \mathrm{Fe}\left(\mathrm{MoO}_{4}\right)_{6}$. It is compared and discussed with similar structures.

\section{Contexte chimique}

Ces dernières années, plusieurs équipes de recherche s'intéressent à l'étude des systèmes quaternaires de type $A-M-\mathrm{Mo}-$ $\mathrm{O}(A=$ cation monovalent et $M=$ métal de transition). Les molybdates présentent plusieurs domaines d'applications: matériaux laser (Khal'baeva et al., 2013; Hanuza \& Maczka, 1994), ferroélectriques (Isupov, 2005; Khal'baeva et al., 2012), piézoélectriques, catalyseurs pour la synthése organique, superionique, liants à haute température, matrices pour la fixation des isotopes à vie longue et autres matériaux (Kotova \& Kozhevnikova, 2003).

Il est nécessaire de noter que les phases rhomboédriques ont un intérêt particulier car elles présentent une cris-

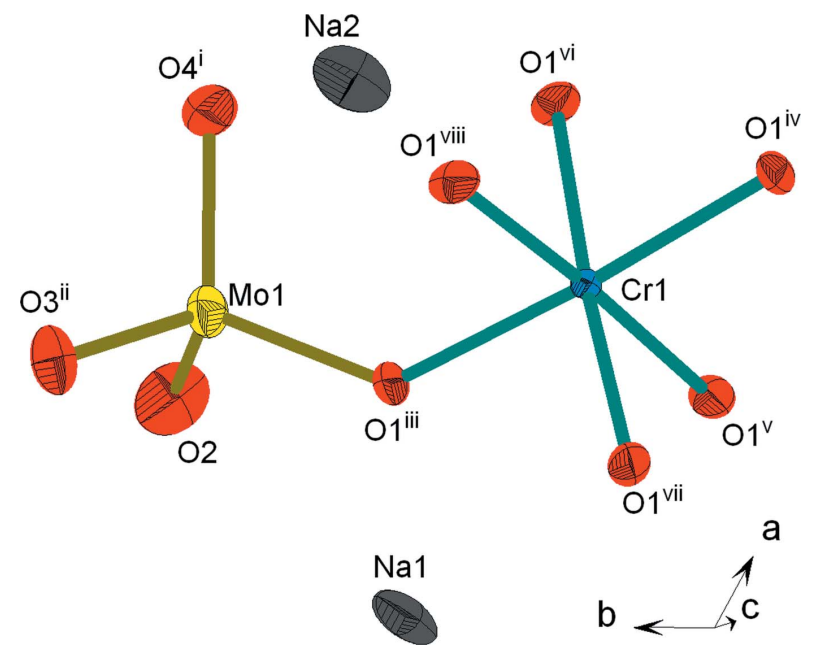

Figure 1

Représentation des polyédres de coordination de l'unité structurale dans $\mathrm{Na}_{9} \mathrm{Cr}\left(\mathrm{MoO}_{4}\right)_{6}$. Les éllipsoïdes ont été définis avec $50 \%$ de probabilité. [Codes de symétrie: (i) $-x+\frac{2}{3},-x+y+\frac{1}{3},-z+\frac{5}{6}$; (ii) $-y+\frac{4}{3},-x+\frac{2}{3}, z-\frac{5}{6}$; (iii) $y-\frac{1}{3}, x+\frac{1}{3},-z+\frac{5}{6}$; (iv) $y-\frac{1}{3}, x+\frac{1}{3},-z+\frac{11}{6}$; (v) $-y+1, x-y+1, z$; (vi) $-x+y,-x+1, z$; (vii) $x-y+\frac{2}{3},-y+\frac{4}{3},-z+\frac{11}{6}$; (viii) $-x+\frac{2}{3},-x+y+\frac{1}{3}$, $-z+\frac{11}{6}$.] 

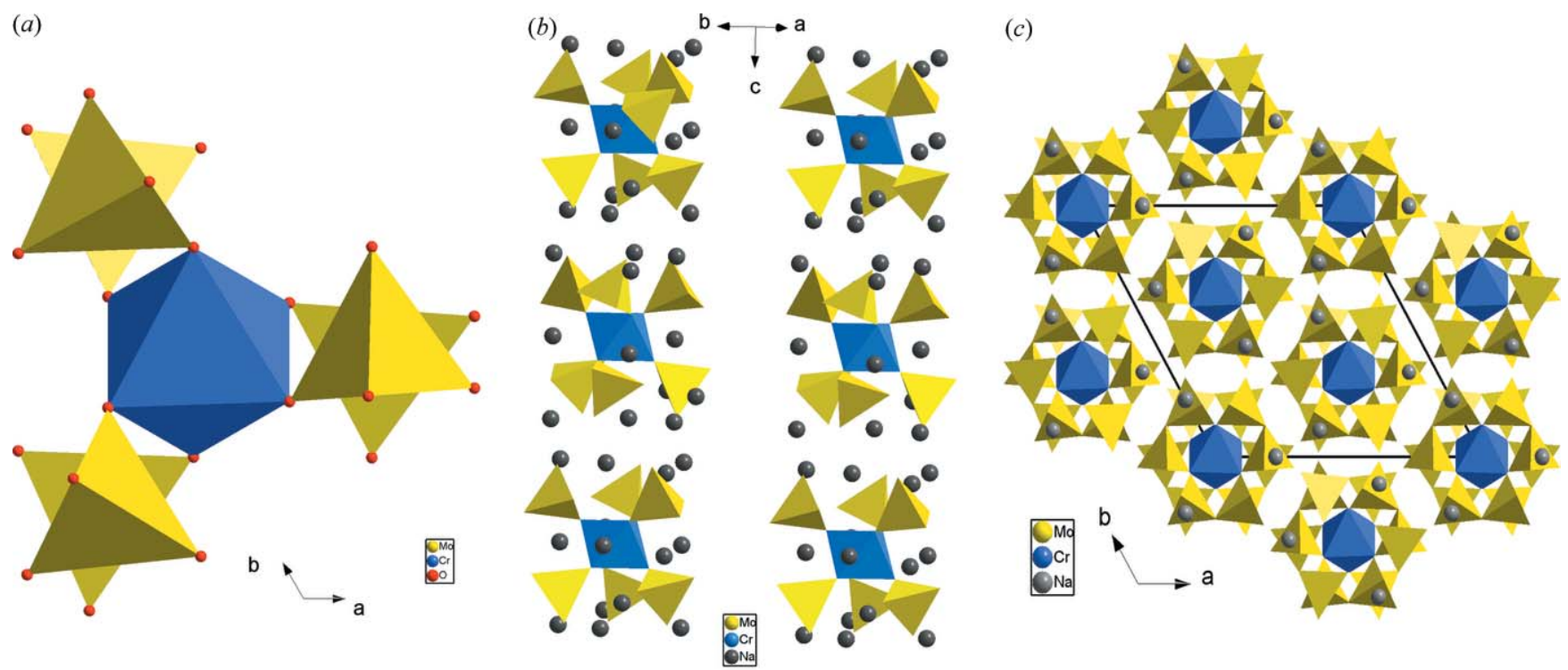

Figure 2

(a) Projection d'un cluster $\left[\mathrm{Cr}\left(\mathrm{MoO}_{4}\right)_{6}\right]^{9-},(b)$ représentation des unités structurales de $\mathrm{Na}_{9} \mathrm{Cr}\left(\mathrm{MoO}_{4}\right)_{6},(c)$ projection de la structure de $\mathrm{Na}_{9} \mathrm{Cr}(\mathrm{MoO})_{6}$ dans le plan (001).

tallochimie similaire avec des composés du type NASICON, qui sont caractérisés par des propriétés superioniques (Kotova \& Kozhevnikova, 2003). C'est dans ce cadre, que nous avons choisi l'exploration des systèmes quaternaires, très peu étudiés à notre connaissance, $A-\mathrm{Cr}-\mathrm{Mo}-\mathrm{O}$ ( $A=$ ion monovalent). Une nouvelle phase de formulation $\mathrm{Na}_{9} \mathrm{Cr}\left(\mathrm{MoO}_{4}\right)_{6}$ a été obtenue par réaction à l'état solide à $798 \mathrm{~K}$.

\section{Commentaire structurelle}

L'unité structurale dans la phase $\mathrm{Na}_{9} \mathrm{Cr}\left(\mathrm{MoO}_{4}\right)_{6}$ est construite à partir d'un octaèdre $\mathrm{CrO}_{6}$ relié par mise en commun d'un sommet à un tétraèdre $\mathrm{MoO}_{4}$. La compensation de charges est

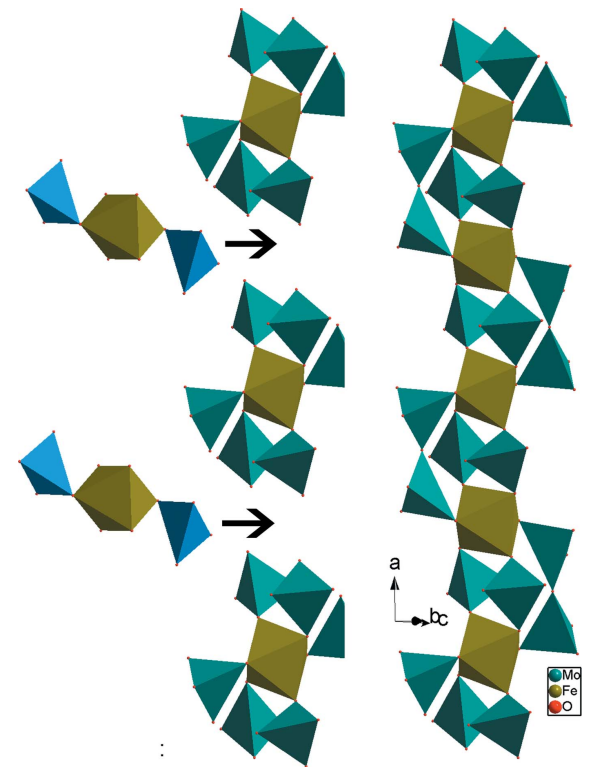

Figure 3

Représentation d'un ruban mettant en évidence la connection entre les unités $\left[\mathrm{Fe}\left(\mathrm{MoO}_{4}\right)_{6}\right]$ de la structure de $\mathrm{Rb}_{3} \mathrm{FeMo}_{4} \mathrm{O}_{15}$. assurée par les cations $\mathrm{Na}^{+}$(Fig. 1). Dans la charpente anionique chaque octaèdre $\mathrm{CrO}_{6}$ partage ses six sommets avec six tétraèdres $\mathrm{MoO}_{4}$ différents pour conduire aux clusters $\left[\mathrm{Cr}\left(\mathrm{MoO}_{4}\right)_{6}\right]^{9-}$ (Fig. 2a). Ces derniers, dirigés selon [001], prennent une disposition laissant libre des espaces où résident les cations $\mathrm{Na}^{+}$(Fig. $2 b$ ). La répartition de l'ensemble de ces unités selon les trois directions de l'espace engendre une charpente anionique zérodimensionnelle (0D) dans laquelle les cations $\mathrm{Na}^{+}$sont orientés vers les sommets libres des tétraèdres $\mathrm{MoO}_{4}$ (Fig. 2c).

L'examen des facteurs géométriques révèle que les distances $\mathrm{Mo}-\mathrm{O}$ et $\mathrm{Cr}-\mathrm{O}$ dans respectivement les tétraèdres $\mathrm{MoO}_{4}$ et les octaèdres $\mathrm{CrO}_{6}$ sont similaires à celles rencontrées dans la littérature (Sarapulova et al., 2009; Tsyrenova et al., 2009; Gicquel-Mayer et al., 1981; Bensaid et al., 2013; Averbuch-Pouchot et al., 1981). En effet, dans le tétraèdre $\mathrm{MoO}_{4}$ les distances $\mathrm{Mo}-\mathrm{O}$ sont situées dans la gamme 1,723 (4)-1,793 (4) A. La plus longue liaison Mo-O correspond à l'oxygène du pont mixte $\mathrm{Cr}-\mathrm{O} 1-\mathrm{Mo}$. Les distances $\mathrm{Cr}-\mathrm{O}$ sont toutes égales à 1,966 (3) Å (tableau 1). Les angles $\mathrm{O}-\mathrm{Mo}-\mathrm{O}$, situés entre $108,2(2)-111,8(2)^{\circ}$ correspondent à des tétraèdres $\mathrm{MoO}_{4}$ presque réguliers.

Tableau 1

Longueurs des liaisons sélectionnées $(\AA)$.

\begin{tabular}{llll}
\hline $\mathrm{Mo} 1-\mathrm{O} 4^{\mathrm{i}}$ & $1.723(4)$ & $\mathrm{Na} 1-\mathrm{O} 4$ & $2.649(4)$ \\
$\mathrm{Mo} 1-\mathrm{O} 2$ & $1.730(4)$ & $\mathrm{Na} 2-\mathrm{O} 2$ & $2.308(4)$ \\
$\mathrm{Mo} 1-3^{\text {ii }}$ & $1.745(4)$ & $\mathrm{Na} 2-\mathrm{O} 2^{\text {iv }}$ & $2.384(5)$ \\
$\mathrm{Mo1}-\mathrm{O} 1^{\mathrm{iii}}$ & $1.793(4)$ & $\mathrm{Na} 2-\mathrm{O} 4^{\mathrm{v}}$ & $2.414(5)$ \\
$\mathrm{Cr} 1-\mathrm{O} 1$ & $1.966(3)$ & $\mathrm{Na} 2-\mathrm{O} 3^{\mathrm{vi}}$ & $2.473(5)$ \\
$\mathrm{Na} 1-\mathrm{O} 1$ & $2.364(4)$ & $\mathrm{Na} 2-\mathrm{O} 3^{\mathrm{v}}$ & $2.559(4)$ \\
$\mathrm{Na} 1-\mathrm{O} 3$ & $2.407(4)$ & $\mathrm{Na} 2-\mathrm{O} 4^{\mathrm{vi}}$ & $2.989(5)$ \\
\hline
\end{tabular}

Symmetry codes: (i) $-x+\frac{2}{3},-x+y+\frac{1}{3},-z+\frac{5}{6}$; (ii) $-y+\frac{4}{3},-x+\frac{2}{3}, z-\frac{5}{6}$; $\quad$ (iii) $y-\frac{1}{3}, x+\frac{1}{3},-z+\frac{5}{6} ; \quad$ (iv) $\quad x-y+\frac{2}{3}, x+\frac{1}{3},-z+\frac{1}{3} ; \quad$ (v) $\quad-x+y, y, z-\frac{1}{2}$; $\quad$ (vi) $y,-x+y,-z+1$. 


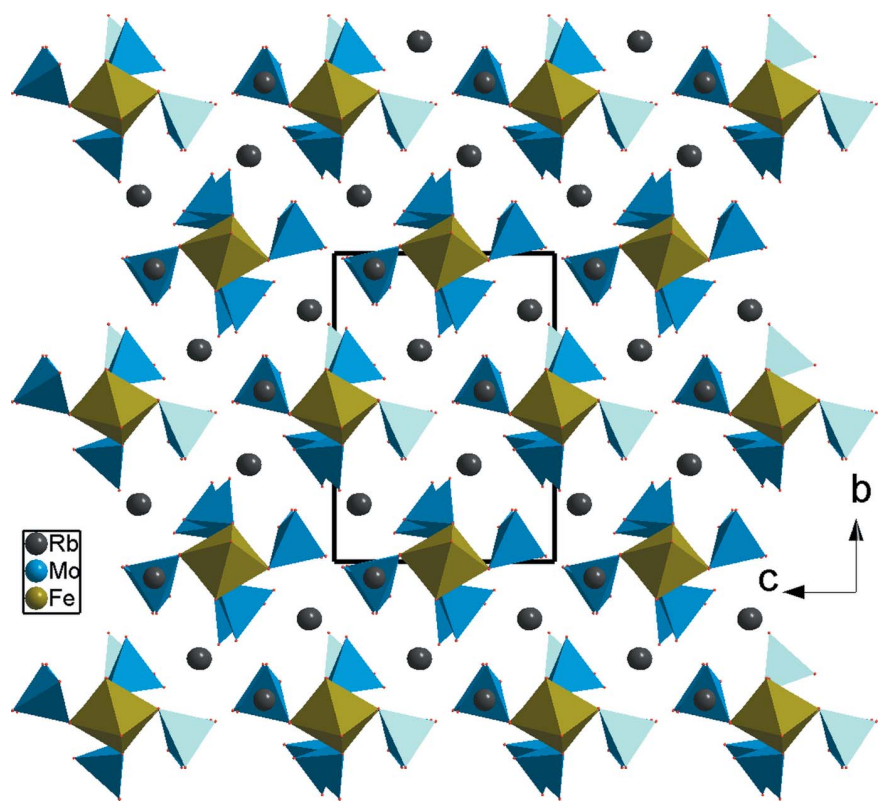

Figure 4

Projection de la structure de $\mathrm{Rb}_{3} \mathrm{FeMo}_{4} \mathrm{O}_{15}$ selon $a$.

De plus, le calcul des sommes de valences de liaison (BVS), utilisant la formule empirique de Brown (2002) conduit aux valeurs des charges des ions suivants: Mo1 (6,166), Cr1 $(3,119), \mathrm{Na} 1(1,021), \mathrm{Na} 2(0,986)$ ce qui confirme les degrés d'oxydation des différents ions existant dans la phase étudiée. Un examen bibliographique montre que le matériau obtenu est isostructural aux composés ayant une formulation analogue $\mathrm{Na}_{9} \mathrm{Sc}\left(\mathrm{MoO}_{4}\right)_{6}$ (Savina, Solodovnikov et al., 2013) et $\mathrm{Na}_{9} \mathrm{Fe}\left(\mathrm{MoO}_{4}\right)_{6}$ (Savina, Morozov et al., 2013). C'est deux derniers cristallisent dans le même système rhomboédrique mais présentent une symétrie plus réduite groupe $R \overline{3}$. En effet, ils possèdent une unité asymétrique différente de celle de notre composé. Elle est construite à partir de trois atomes de sodium et d'un octaèdre $M_{6}(M=\mathrm{Sc}, \mathrm{Fe})$ qui est relié par mise en commun des sommets à deux tétraèdres $\mathrm{MoO}_{4}$ différents. La jonction de ces unités par partage de sommets conduit à une charpente similaire à celle du composé obtenu.

\section{Enquête de base de données}

La comparaison de la structure de $\mathrm{Na}_{9} \mathrm{Cr}\left(\mathrm{MoO}_{4}\right)_{6}$ avec celles rencontrées dans la littérature montre une certaine analogie avec les composés $\mathrm{Rb}_{3} \mathrm{FeMo}_{4} \mathrm{O}_{15}$ qui cristallise dans le système monoclinique groupe d'espace $P 2_{1} / c$ (Khal'baeva et al., 2010), $\mathrm{Na}_{3,5} \mathrm{Cr}_{1,83}\left(\mathrm{AsO}_{4}\right)_{3}$ (Fakhar Bourguiba et al., 2013) et $\mathrm{Na}_{3} \mathrm{Cr}_{2}\left(\mathrm{PO}_{4}\right)_{3}$ (Genkina et al., 1991) cristallisant dans le même groupe d'espace $R \overline{3} c$. Le composé $\mathrm{Rb}_{3} \mathrm{FeMo}_{4} \mathrm{O}_{15}$ est formé par des clusters $\left[\mathrm{Fe}\left(\mathrm{MoO}_{4}\right)_{6}\right]^{9-}$ ayant le même arrangement des polyèdres que celui du cluster $\left[\mathrm{Cr}\left(\mathrm{MoO}_{4}\right)_{6}\right]^{9-}$ existant dans notre structure. De plus, dans $\mathrm{Rb}_{3} \mathrm{Fe}\left(\mathrm{MoO}_{4}\right)_{2} \mathrm{Mo}_{2} \mathrm{O}_{7}$, les deux clusters sont liés à l'aide d'une unité linéaire $\mathrm{FeMo}_{2} \mathrm{O}_{12}$ moyennant des ponts mixtes de type Mo-O-Fe et aussi des ponts simples Mo-O-Mo faisant apparaître des groupements dimolybdates $\mathrm{Mo}_{2} \mathrm{O}_{7}$ (Fig. 3). Il

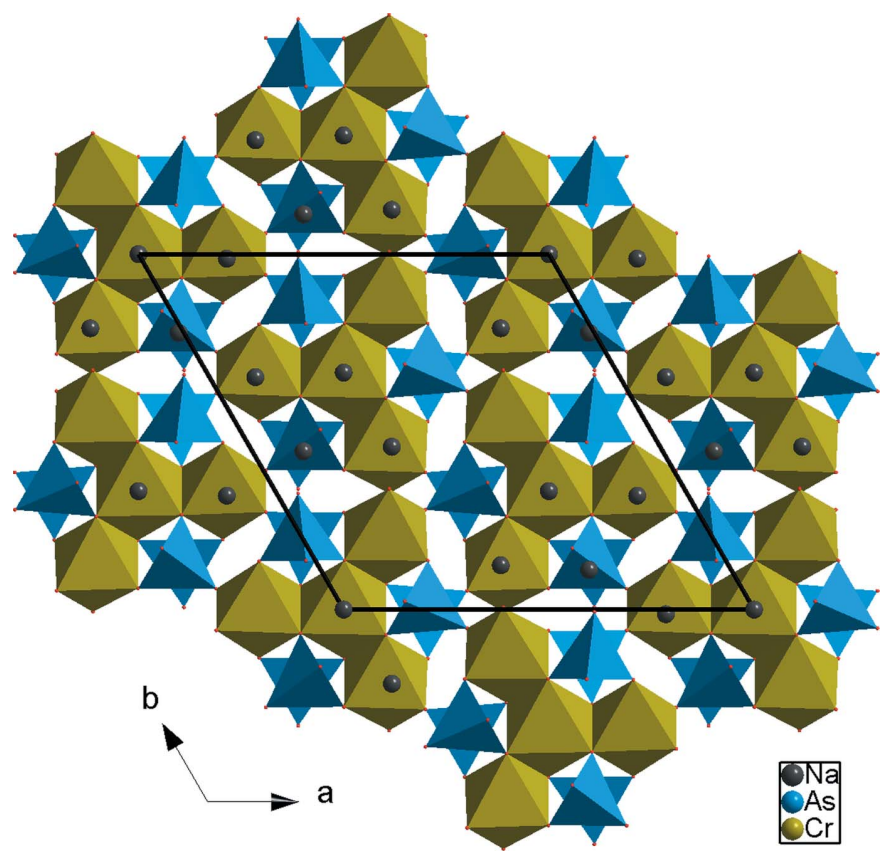

Figure 5

Présentation, selon [001], du réseau tridimensionnel du composé $\mathrm{Na}_{3,5} \mathrm{Cr}_{1,83}\left(\mathrm{AsO}_{4}\right)_{3}$.

en résulte, des rubans disposés parallèlement à la direction [100] qui présente à leur tour une charpente unidimensionnelle possédant des espaces inter-rubans où résident les cations monovalents $\mathrm{Rb}^{+}$(Fig. 4). Un examen de la charpente anionique du composé $\mathrm{Na}_{3,5} \mathrm{Cr}_{1,83}\left(\mathrm{AsO}_{4}\right)_{3}$ révèle la présence des unités formulaires $\left[\mathrm{Cr}_{4}\left(\mathrm{AsO}_{4}\right)_{6}\right]^{6-}$. En effet, elles sont formées par des unités $\left[\mathrm{Cr} 1\left(\mathrm{AsO}_{4}\right)_{6}\right]$ similaires à celles de notre phase mais dans laquelle chaque octaèdre central $\mathrm{Cr} \mathrm{O}_{6}$ est lié, en plus de partage d'arêtes, à respectivement trois octaèdres $\mathrm{CrO}_{6}$ différents. Ces unités se connectent moyennant des ponts mixtes de type As-O-Cr formant ainsi un réseau tridimensionnel (Fig. 5). La charpente anionique du composé $\mathrm{Na}_{3} \mathrm{Cr}_{2}\left(\mathrm{PO}_{4}\right)_{3}$ présente des unités de type $\left[\mathrm{Cr}\left(\mathrm{PO}_{4}\right)_{6}\right]^{15-}$ possédant une disposition de polyèdres similaire à celle de notre phase. Dans le phosphate $\mathrm{Na}_{3} \mathrm{Cr}_{2}\left(\mathrm{PO}_{4}\right)_{3}$, chaque unité se lie par formation de ponts mixtes $\mathrm{P}-\mathrm{O}-\mathrm{Cr}$ avec les unités voisines pour conduire à une charpente tridimensionnelle (Fig. 6).

\section{Synthèse et cristallisation}

Les cristaux relatifs à $\mathrm{Na}_{9} \mathrm{Cr}\left(\mathrm{MoO}_{4}\right)_{6}$ ont été obtenus par réaction à l'état solide à partir des réactifs: $\mathrm{Na}_{2} \mathrm{CO}_{3}$ (PROLABO, 70128, 99,6\%), $\mathrm{Cr}\left(\mathrm{NO}_{3}\right)_{3} \cdot 9 \mathrm{H}_{2} \mathrm{O}$ (FLUKA, $60832,99,0 \%)$ et $\left(\mathrm{NH}_{4}\right)_{6} \mathrm{Mo}_{7} \mathrm{O}_{24} \cdot 4 \mathrm{H}_{2} \mathrm{O}$ (SIGMA-ALDRICH, $13301,99,0 \%)$ pris dans les proportions telques $\mathrm{Na}: \mathrm{Cr}: \mathrm{Mo}=$ 9:0,5:6. Après un broyage effectué dans un mortier en agate, le mélange a été mis dans un creuset en porcelaine, puis préchauffé à l'air à $453 \mathrm{~K}$ pendant 24 heures en vue d'éliminer les composés volatils. Il est ensuite porté jusqu'à une température voisine de celle de la fusion à $798 \mathrm{~K}$. Le mélange est abandonné à cette température pendant quelques jours 


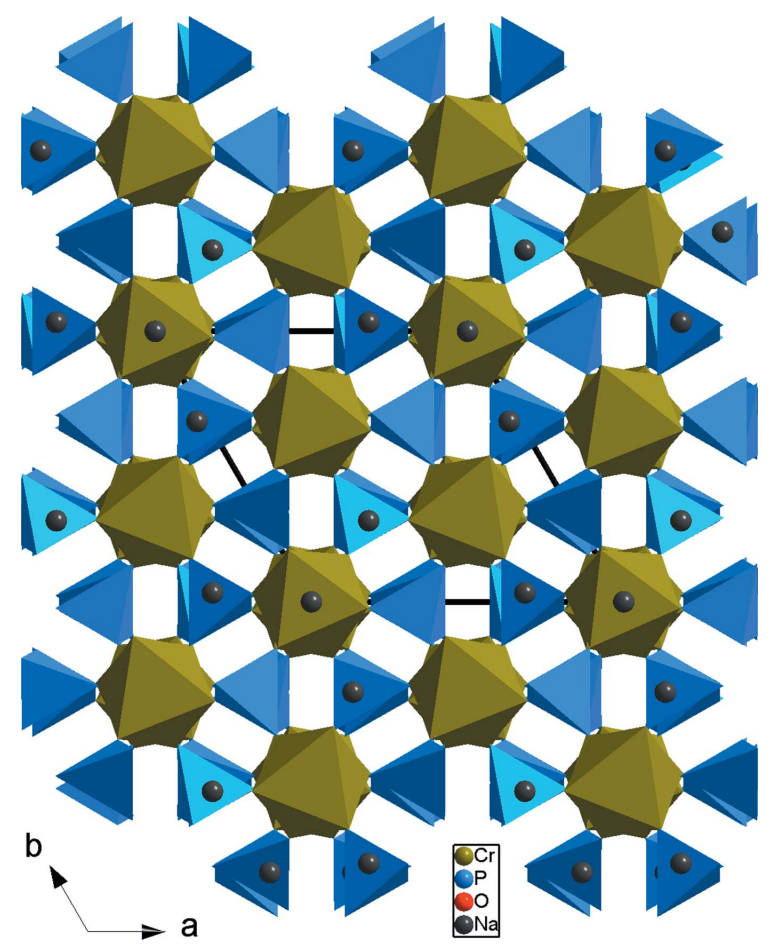

Figure 6

Projection, selon $c$, de la structure du $\mathrm{Na}_{3} \mathrm{Cr}_{2}\left(\mathrm{PO}_{4}\right)_{3}$.

pour favoriser la germination et la croissance des cristaux. Par la suite, il a subi en premier lieu un refroidissement lent $\left(5^{\circ} /\right.$ demi journée) jusqu'à $748 \mathrm{~K}$ puis rapide $\left(50^{\circ} / \mathrm{h}\right)$ jusqu'à la température ambiante. Des cristaux de couleur rouge de taille suffisante pour les mesures des intensités sont obtenus. La morphologie et l'analyse élementaire, ont été faites au moyent d'un microscope électronique à balayage de type FEI QUANTA 200. Cette analyse confirme la présence des éléments attendus: $\mathrm{Na}, \mathrm{Cr}$, Mo et l'oxyène (Fig. 7).

\section{Affinement}

Les conditions expérimentales de la collecte des données et le résultat final de l'affinement sont rassemblés dans le tableau 2.

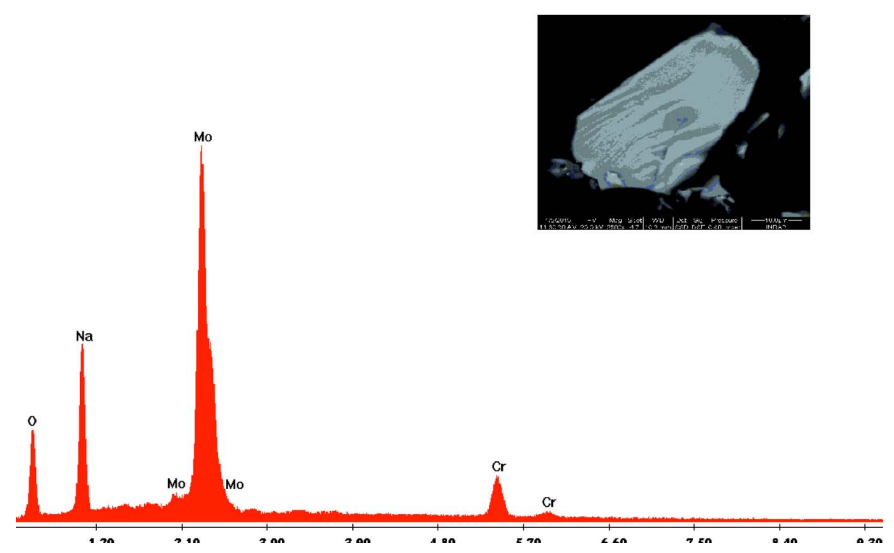

Figure 7

Morphologie et analyse qualitative d'un cristal de $\mathrm{Na}_{9} \mathrm{Cr}\left(\mathrm{MoO}_{4}\right)_{6}$.
Tableau 2

Détails expérimentaux.

\begin{tabular}{|c|c|}
\hline \multicolumn{2}{|l|}{ Données crystallines } \\
\hline Formule chimique & $\mathrm{Na}_{9} \mathrm{Cr}\left(\mathrm{MoO}_{4}\right)_{6}$ \\
\hline$M_{\mathrm{r}}$ & 1218,55 \\
\hline Système cristallin, groupe d'espace & Trigonal, $R \overline{3} c$ \\
\hline Température (K) & 298 \\
\hline$a, c(\AA)$ & $14,707(5), 19,175(7)$ \\
\hline$V\left(\AA^{3}\right)$ & $3592(2)$ \\
\hline$Z$ & 6 \\
\hline Type de rayonnement & Мо $K \alpha$ \\
\hline$\mu\left(\mathrm{mm}^{-1}\right)$ & 3,74 \\
\hline Taille des cristaux (mm) & $0,22 \times 0,16 \times 0,10$ \\
\hline \multicolumn{2}{|l|}{ Collection de données } \\
\hline Diffractomètre & Enraf-Nonius CAD-4 \\
\hline Correction d'absorption & $\psi$ scan (North et al., 1968) \\
\hline$T_{\min }, T_{\max }$ & $0,481,0,676$ \\
\hline $\begin{array}{l}\text { Nombre de réflexions mesurées, } \\
\text { indépendantes et observées } \\
{[I>2 \sigma(I)]}\end{array}$ & $2549,875,678$ \\
\hline$R_{\text {int }}$ & 0,065 \\
\hline$(\sin \theta / \lambda)_{\max }\left(\AA^{-1}\right)$ & 0,638 \\
\hline \multicolumn{2}{|l|}{ Affinement } \\
\hline$R\left[F^{2}>2 \sigma\left(F^{2}\right)\right], w R\left(F^{2}\right), S$ & $0,028,0,078,1,13$ \\
\hline Nombre de réflexions & 875 \\
\hline Nombre de paramètres & 63 \\
\hline$\Delta \rho_{\max }, \Delta \rho_{\min }\left(\mathrm{e} \AA^{-3}\right)$ & $0,60,-0,57$ \\
\hline
\end{tabular}

Programmes informatiques: CAD-4 EXPRESS (Duisenberg, 1992; Macíček \& Yordanov, 1992), XCAD4 (Harms \& Wocadlo, 1995), SHELXS97 et SHELXL97 (Sheldrick, 2008), DIAMOND (Brandenburg \& Putz, 1999) et WinGX (Farrugia, 2012).

En effet, la structure a été résolue et affinée, sans ambiguïté dans le groupe d'espace $R \overline{3} c$ par les méthodes directes utilisant la chaíne de programmes SHELXS97 (Sheldrick, 2008) inclue dans le système Win $G X$ publication (Farrugia, 2012), partant de la formule $\mathrm{Na}_{3} \mathrm{Cr}_{1} \mathrm{Mo}_{3} \mathrm{O}_{12}$. Les premiers cycles d'affinements ont permis de localiser la plupart des atomes. Un examen de la Fourier différence et en se basant sur des considérations géométriques et de neutralité électrique, l'un des atomes d'oxygène a été remplacé par un sodium. L'affinement de tous les paramètres variables conduit à des ellipsoïdes bien définis. Les densités d'électrons maximum et minimum restants dans la Fourier-différence finale sont acceptables et sont situées respectivement à $0,70 \AA$ A de $\mathrm{O} 4$ et à 1,06 ̊̊ de Mo1.

\section{Remerciements}

Les auteurs remercient le Ministère de l'Enseignement Supérieur, de la Recherche Scientifique et de la Technologie de la Tunisie pour le financement du laboratoire LMC (code LR01ES11).

\section{Références}

Averbuch-Pouchot, M. T., Durif, A. \& Guitel, J. C. (1981). J. Solid State Chem. 38, 253-258.

Bensaid, H., El Bouari, A., Benmokhtar, S., Manoun, B., Bih, L. \& Lazor, P. (2013). J. Mol. Struct. 1031, 152-159.

Brandenburg, K. \& Putz, H. (1999). DIAMOND. Crystal Impact GbR, Bonn, Allemagne. 
Brown, I. D. (2002). The Chemical Bond in Inorganic Chemistry. The Bond Valence Model. Oxford University Press.

Duisenberg, A. J. M. (1992). J. Appl. Cryst. 25, 92-96.

Fakhar Bourguiba, N., Ouerfelli, N. \& Zid, M. F. (2013). J. Adv. Chem. 10, 3160-3170.

Farrugia, L. J. (2012). J. Appl. Cryst. 45, 849-854.

Genkina, E. A., Kalinin, V. B., Maksimov, B. A. \& Golubev, A. M. (1991). Kristallografiya, 36, 1126-1130.

Gicquel-Mayer, C., Mayer, M. \& Pérez, G. (1981). Acta Cryst. B37, 1035-1039.

Hanuza, J. \& Maczka, M. (1994). Vib. Spectrosc. 7, 85-96.

Harms, K. \& Wocadlo, S. (1995). XCAD4. Université de Marburg, Allemagne.

Isupov, V. A. (2005). Ferroelectrics, 321, 63-90.

Khal'baeva, K. M., Solodovnikov, S. F., Khaikina, E. G., Kadyrova, Y. M., Solodovnikova, Z. A. \& Basovich, O. M. (2010). J. Solid State Chem. 183, 712-719.

Khal'baeva, K. M., Solodovnikov, S. F., Khaikina, E. G., Kadyrova, Y. M., Solodovnikova, Z. A. \& Basovich, O. M. (2012). J. Solid State Chem. 187, 276-281.
Khal'baeva, K. M., Solodovnikov, S. F., Khaikina, E. G., Kadyrova, Y. M., Solodovnikova, Z. A. \& Basovich, O. M. (2013). J. Solid State Chem. 203, 227-231.

Kotova, I. Yu. \& Kozhevnikova, N. M. (2003). Russ. J. Appl. Chem. 76, 1572-1576.

Macíček, J. \& Yordanov, A. (1992). J. Appl. Cryst. 25, 73-80.

North, A. C. T., Phillips, D. C. \& Mathews, F. S. (1968). Acta Cryst. A24, 351-359.

Sarapulova, A., Mikhailova, D., Senyshyn, A. \& Ehrenberg, H. (2009). J. Solid State Chem. 182, 3262-3268.

Savina, A. A., Morozov, V. A., Basovich, O. M., Khaikina, E. G. \& Lazoryak, B. I. (2013). Acta Cryst. C69, 1301-1303.

Savina, A. A. F., Solodovnikov, S. M., Basovich, O. A., Solodovnikova, Z. A., Belov, D. V., Pokholok, K. A., Gudkova, I., Stefanovich, S. Y. I., Lazoryak, B. \& Khaikina, E. G. (2013). J. Solid State Chem. 205, 149-153.

Sheldrick, G. M. (2008). Acta Cryst. A64, 112-122.

Tsyrenova, G. D., Solodovnikov, S. F., Pavlova, E. T., Khaikina, E. G. \& Solodovnikova, Z. A. (2009). Russ. J. Inorg. Chem. 54, 743-750. 


\section{supporting information}

Acta Cryst. (2015). E71, 435-439 [https://doi.org/10.1107/S2056989015005976]

Synthèse et étude structurale de $\mathrm{Na}_{9} \mathrm{Cr}\left(\mathrm{MoO}_{4}\right)_{6}$

Wassim Dridi, Ines Ennajeh et Mohamed Faouzi Zid

Computing details

Data collection: CAD-4 EXPRESS (Duisenberg, 1992; Macíček \& Yordanov, 1992); cell refinement: CAD-4 EXPRESS (Duisenberg, 1992; Macíček \& Yordanov, 1992); data reduction: XCAD4 (Harms \& Wocadlo, 1995); program(s) used to solve structure: SHELXS97 (Sheldrick, 2008); program(s) used to refine structure: SHELXL97 (Sheldrick, 2008); molecular graphics: DIAMOND (Brandenburg \& Putz, 1999); software used to prepare material for publication: WinGX (Farrugia, 2012).

Nonasodium chromium(III) hexakis[molybdate(VI)]

\section{Crystal data}

$\mathrm{Na}_{9} \mathrm{Cr}\left(\mathrm{MoO}_{4}\right)_{6}$

$M_{r}=1218.55$

Trigonal, $R \overline{3} c$

Hall symbol: -R 32 "c

$a=14.707(5) \AA$

$c=19.175(7) \AA$

$V=3592(2) \AA^{3}$

$Z=6$

$F(000)=3402$

Data collection

Enraf-Nonius CAD-4 diffractometer

Radiation source: fine-focus sealed tube

Graphite monochromator

$\omega / 2 \theta$ scans

Absorption correction: $\psi$ scan

(North et al., 1968)

$T_{\min }=0.481, T_{\max }=0.676$

2549 measured reflections

\section{Refinement}

Refinement on $F^{2}$

Least-squares matrix: full

$R\left[F^{2}>2 \sigma\left(F^{2}\right)\right]=0.028$

$w R\left(F^{2}\right)=0.078$

$S=1.13$

875 reflections

63 parameters

0 restraints

Primary atom site location: structure-invariant direct methods
$D_{\mathrm{x}}=3.380 \mathrm{Mg} \mathrm{m}^{-3}$

Mo $K \alpha$ radiation, $\lambda=0.71073 \AA$

Cell parameters from 25 reflections

$\theta=10-15^{\circ}$

$\mu=3.74 \mathrm{~mm}^{-1}$

$T=298 \mathrm{~K}$

Prism, red

$0.22 \times 0.16 \times 0.10 \mathrm{~mm}$

875 independent reflections

678 reflections with $I>2 \sigma(I)$

$R_{\text {int }}=0.065$

$\theta_{\max }=27.0^{\circ}, \theta_{\min }=2.7^{\circ}$

$h=-16 \rightarrow 4$

$k=-17 \rightarrow 1$

$l=-24 \rightarrow 24$

2 standard reflections every 2 reflections

intensity decay: $1.2 \%$

Secondary atom site location: difference Fourier

map

$w=1 /\left[\sigma^{2}\left(F_{\mathrm{o}}{ }^{2}\right)+(0.0286 P)^{2}+21.2743 P\right]$

where $P=\left(F_{\mathrm{o}}^{2}+2 F_{\mathrm{c}}^{2}\right) / 3$

$(\Delta / \sigma)_{\max }<0.001$

$\Delta \rho_{\max }=0.60$ e $\AA^{-3}$

$\Delta \rho_{\min }=-0.57$ e $\AA^{-3}$

Extinction correction: SHELXL, $\mathrm{Fc}^{*}=\mathrm{kFc}\left[1+0.001 \mathrm{xFc}^{2} \lambda^{3} / \sin (2 \theta)\right]^{-1 / 4}$

Extinction coefficient: 0.00013 (4) 


\section{Special details}

Geometry. All e.s.d.'s (except the e.s.d. in the dihedral angle between two l.s. planes) are estimated using the full covariance matrix. The cell e.s.d.'s are taken into account individually in the estimation of e.s.d.'s in distances, angles and torsion angles; correlations between e.s.d.'s in cell parameters are only used when they are defined by crystal symmetry. An approximate (isotropic) treatment of cell e.s.d.'s is used for estimating e.s.d.'s involving 1.s. planes.

Refinement. Refinement of $F^{2}$ against ALL reflections. The weighted $R$-factor $w R$ and goodness of fit $S$ are based on $F^{2}$, conventional $R$-factors $R$ are based on $F$, with $F$ set to zero for negative $F^{2}$. The threshold expression of $F^{2}>\sigma\left(F^{2}\right)$ is used only for calculating $R$-factors (gt) etc. and is not relevant to the choice of reflections for refinement. $R$-factors based on $F^{2}$ are statistically about twice as large as those based on $F$, and $R$ - factors based on ALL data will be even larger.

Fractional atomic coordinates and isotropic or equivalent isotropic displacement parameters $\left(\hat{A}^{2}\right)$

\begin{tabular}{lllll}
\hline & $x$ & $y$ & $z$ & $U_{\text {iso }} * U_{\text {eq }}$ \\
\hline Mo1 & $0.51969(4)$ & $0.66053(4)$ & $0.02393(2)$ & $0.01759(19)$ \\
Cr1 & 0.3333 & 0.6667 & 0.9167 & $0.0096(4)$ \\
Na1 & $0.1089(2)$ & 0.6667 & 0.9167 & $0.0285(8)$ \\
Na2 & $0.5617(2)$ & $0.6706(2)$ & $0.21706(11)$ & $0.0334(6)$ \\
O1 & $0.2729(3)$ & $0.7346(3)$ & $0.85988(19)$ & $0.0177(8)$ \\
O2 & $0.4881(4)$ & $0.6129(4)$ & $0.1082(2)$ & $0.0342(11)$ \\
O3 & $0.0440(4)$ & $0.7277(3)$ & $0.8230(2)$ & $0.0267(9)$ \\
O4 & $0.0834(3)$ & $0.5458(4)$ & $0.8075(2)$ & $0.0312(10)$
\end{tabular}

Atomic displacement parameters $\left(\AA^{2}\right)$

\begin{tabular}{lllllll}
\hline & $U^{11}$ & $U^{22}$ & $U^{33}$ & $U^{12}$ & $U^{13}$ & $U^{23}$ \\
\hline Mo1 & $0.0165(3)$ & $0.0218(3)$ & $0.0164(3)$ & $0.0110(2)$ & $-0.00230(17)$ & $0.00171(18)$ \\
Cr1 & $0.0070(5)$ & $0.0070(5)$ & $0.0149(8)$ & $0.0035(3)$ & 0.000 & 0.000 \\
Na1 & $0.0211(11)$ & $0.049(2)$ & $0.0250(15)$ & $0.0244(12)$ & $0.0073(8)$ & $0.0146(16)$ \\
Na2 & $0.0341(14)$ & $0.0518(18)$ & $0.0200(11)$ & $0.0258(14)$ & $-0.0021(10)$ & $-0.0027(11)$ \\
O1 & $0.0142(18)$ & $0.0155(19)$ & $0.0246(18)$ & $0.0083(15)$ & $-0.0003(15)$ & $0.0083(15)$ \\
O2 & $0.037(3)$ & $0.041(3)$ & $0.0153(18)$ & $0.012(2)$ & $-0.0028(18)$ & $0.0009(19)$ \\
O3 & $0.037(3)$ & $0.024(2)$ & $0.028(2)$ & $0.0221(19)$ & $-0.0003(19)$ & $-0.0009(19)$ \\
O4 & $0.025(2)$ & $0.031(2)$ & $0.043(2)$ & $0.018(2)$ & $-0.0141(19)$ & $-0.014(2)$ \\
\hline
\end{tabular}

Geometric parameters $\left(A,{ }^{\circ}\right)$

\begin{tabular}{llll}
\hline $\mathrm{Mo} 1-\mathrm{O} 4^{\mathrm{i}}$ & $1.723(4)$ & $\mathrm{Na} 1-\mathrm{O} 1$ & $2.364(4)$ \\
$\mathrm{Mo} 1-\mathrm{O} 2$ & $1.730(4)$ & $\mathrm{Na} 1-\mathrm{O} 3$ & $2.407(4)$ \\
$\mathrm{Mo} 1-\mathrm{O} 3^{\text {ii }}$ & $1.745(4)$ & $\mathrm{Na} 1-\mathrm{O} 3^{\text {vi }}$ & $2.407(4)$ \\
$\mathrm{Mo1} 1-\mathrm{O} 1^{\text {iii }}$ & $1.793(4)$ & $\mathrm{Na} 1-\mathrm{O} 4$ & $2.649(4)$ \\
$\mathrm{Cr} 1-\mathrm{O} 1^{\text {iv }}$ & $1.966(3)$ & $\mathrm{Na} 1-\mathrm{O} 4^{\text {vi }}$ & $2.649(4)$ \\
$\mathrm{Cr} 1-\mathrm{O} 1$ & $1.966(3)$ & $\mathrm{Na} 2-\mathrm{O} 2$ & $2.308(4)$ \\
$\mathrm{Cr} 1-\mathrm{O} 1^{\mathrm{v}}$ & $1.966(3)$ & $\mathrm{Na} 2-\mathrm{O} 2^{\text {ix }}$ & $2.384(5)$ \\
$\mathrm{Cr} 1-\mathrm{O} 1^{\text {vi }}$ & $1.966(3)$ & $\mathrm{Na} 2-\mathrm{O} 4^{\mathrm{x}}$ & $2.414(5)$ \\
$\mathrm{Cr} 1-\mathrm{O} 1^{\text {vii }}$ & $1.966(3)$ & $\mathrm{Na} 2-\mathrm{O} 3^{\text {xi }}$ & $2.473(5)$ \\
$\mathrm{Cr} 1-\mathrm{O} 1^{\text {viii }}$ & $1.966(3)$ & $\mathrm{Na} 2-\mathrm{O} 3^{\mathrm{x}}$ & $2.559(4)$ \\
$\mathrm{Na} 1-\mathrm{O} 1^{\text {vii }}$ & $2.364(4)$ & $\mathrm{Na} 2-\mathrm{O} 4^{\text {xi }}$ & $2.989(5)$ \\
& & & \\
$\mathrm{O} 4-\mathrm{Mo} 1-\mathrm{O} 2$ & $109.2(2)$ & $\mathrm{O} 3-\mathrm{Na} 1-\mathrm{O} 4^{\text {vii }}$ & $120.99(15)$
\end{tabular}




$$
\begin{aligned}
& \mathrm{O} 4^{\mathrm{i}}-\mathrm{Mo} 1-\mathrm{O}^{\mathrm{ii}} \\
& \mathrm{O} 2-\mathrm{Mo} 1-\mathrm{O}^{\mathrm{ii}} \\
& \mathrm{O} 4-\mathrm{Mo} 1-\mathrm{O} 1^{\mathrm{iii}} \\
& \mathrm{O} 2-\mathrm{Mo1}-\mathrm{O}{ }^{\mathrm{iii}}
\end{aligned}
$$

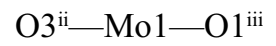

$$
\begin{aligned}
& \mathrm{O}^{\mathrm{iv}}-\mathrm{Cr} 1-\mathrm{O} 1 \\
& \mathrm{O}^{\mathrm{iv}}-\mathrm{Cr} 1-\mathrm{O}^{\mathrm{v}} \\
& \mathrm{O} 1-\mathrm{Cr} 1-\mathrm{O}^{\mathrm{v}} \\
& \mathrm{O} 1^{\mathrm{iv}}-\mathrm{Cr} 1-\mathrm{O}^{\mathrm{vi}} \\
& \mathrm{O} 1-\mathrm{Cr} 1-\mathrm{O}^{\mathrm{vi}} \\
& \mathrm{O} 1^{\mathrm{v}}-\mathrm{Cr} 1-\mathrm{O}^{\mathrm{vi}} \\
& \mathrm{O}^{\mathrm{iv}}-\mathrm{Cr} 1-\mathrm{O}^{\mathrm{vii}} \\
& \mathrm{O} 1-\mathrm{Cr} 1-\mathrm{O}^{\mathrm{vii}} \\
& \mathrm{O} 1^{\mathrm{v}}-\mathrm{Cr} 1-\mathrm{O} 1^{\mathrm{vii}} \\
& \mathrm{O} 1^{\text {vi }}-\mathrm{Cr} 1-\mathrm{O} 1^{\text {vii }} \\
& \mathrm{O} 1^{\text {iv }}-\mathrm{Cr} 1-\mathrm{O} 1^{\text {viii }} \\
& \mathrm{O} 1-\mathrm{Cr} 1-\mathrm{O} 1^{\text {viii }} \\
& \mathrm{O} 1^{\mathrm{v}}-\mathrm{Cr} 1-\mathrm{O}^{\mathrm{viii}} \\
& \mathrm{O} 1^{\mathrm{vi}}-\mathrm{Cr} 1-\mathrm{O} 1^{\text {viii }} \\
& \mathrm{O} 1^{\mathrm{vii}}-\mathrm{Cr} 1-\mathrm{O}^{\text {viii }} \\
& \mathrm{O} 1^{\text {vii-Na1-O1 }}
\end{aligned}
$$

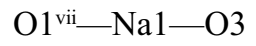

$$
\begin{aligned}
& \text { O1-Na1-O3 }
\end{aligned}
$$

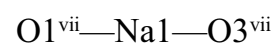

$$
\begin{aligned}
& \mathrm{O} 1-\mathrm{Na} 1-\mathrm{O}^{\text {vii }} \\
& \mathrm{O} 3-\mathrm{Na} 1-\mathrm{O}^{\text {vii }}
\end{aligned}
$$

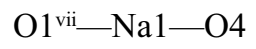

$$
\begin{aligned}
& \mathrm{O} 1-\mathrm{Na} 1-\mathrm{O} 4 \\
& \text { O3-Na1-O4 } \\
& \mathrm{O}^{\text {vii_- }}{ }^{\mathrm{Na} 1}-\mathrm{O} 4
\end{aligned}
$$

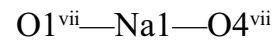

$$
\begin{aligned}
& \mathrm{O} 1-\mathrm{Na} 1-\mathrm{O} 4^{\mathrm{vii}}
\end{aligned}
$$
$108.3(2)$
$108.2(2)$
$111.80(18)$
$108.64(19)$
$110.60(18)$
$176.8(2)$
$90.1(2)$
$92.29(15)$
85.4 (2)
$92.29(15)$
$92.29(15)$

\begin{tabular}{|c|c|}
\hline $\mathrm{O} 3^{\text {vii }}-\mathrm{Na} 1-\mathrm{O} 4^{\text {vii }}$ & $73.23(14)$ \\
\hline $\mathrm{O} 4-\mathrm{Na} 1-\mathrm{O} 4^{\mathrm{vii}}$ & $157.6(2)$ \\
\hline $\mathrm{O} 2-\mathrm{Na} 2-\mathrm{O} 2^{\mathrm{ix}}$ & $98.5(2)$ \\
\hline $\mathrm{O} 2-\mathrm{Na} 2-\mathrm{O}^{x}$ & $112.11(19)$ \\
\hline $\mathrm{O} 2^{\mathrm{ix}}-\mathrm{Na} 2-\mathrm{O} 4^{\mathrm{x}}$ & $110.62(17)$ \\
\hline $\mathrm{O} 2-\mathrm{Na} 2-\mathrm{O} 3^{\mathrm{xi}}$ & $90.60(17)$ \\
\hline $\mathrm{O} 2^{\mathrm{ix}}-\mathrm{Na} 2-\mathrm{O} 3^{\mathrm{xi}}$ & $121.52(18)$ \\
\hline $\mathrm{O} 4^{\mathrm{x}}-\mathrm{Na} 2-\mathrm{O} 3^{\mathrm{xi}}$ & $118.75(17)$ \\
\hline $\mathrm{O} 2-\mathrm{Na} 2-\mathrm{O} 3^{\mathrm{x}}$ & $164.06(19)$ \\
\hline $\mathrm{O} 2^{\mathrm{ix}}-\mathrm{Na} 2-\mathrm{O} 3^{\mathrm{x}}$ & $92.00(17)$ \\
\hline $\mathrm{O} 4^{\mathrm{x}}-\mathrm{Na} 2-\mathrm{O} 3^{\mathrm{x}}$ & $74.78(15)$ \\
\hline $\mathrm{O} 3^{\mathrm{xi}}-\mathrm{Na} 2-\mathrm{O}^{\mathrm{x}}$ & $73.64(17)$ \\
\hline $\mathrm{O} 2-\mathrm{Na} 2-\mathrm{O} 4^{\mathrm{xi}}$ & $72.48(16)$ \\
\hline $\mathrm{O} 2^{\mathrm{ix}}-\mathrm{Na} 2-\mathrm{O} 4^{\mathrm{xi}}$ & $168.70(19)$ \\
\hline $\mathrm{O} 4^{\mathrm{x}}-\mathrm{Na} 2-\mathrm{O} 4^{\mathrm{xi}}$ & $68.09(17)$ \\
\hline $\mathrm{O} 3^{\mathrm{xi}}-\mathrm{Na} 2-\mathrm{O} 4^{\mathrm{xi}}$ & $66.40(14)$ \\
\hline $\mathrm{O} 3^{\mathrm{x}}-\mathrm{Na} 2-\mathrm{O} 4^{\mathrm{xi}}$ & $98.30(16)$ \\
\hline $\mathrm{Mo} 1-\mathrm{O} 2-\mathrm{Na} 2$ & $135.6(3)$ \\
\hline $\mathrm{Mo} 1-\mathrm{O} 2-\mathrm{Na} 2^{\mathrm{xii}}$ & $114.0(2)$ \\
\hline $\mathrm{Na} 2-\mathrm{O} 2-\mathrm{Na} 2^{\mathrm{xii}}$ & $110.28(17)$ \\
\hline $\mathrm{Mo1}^{\mathrm{xiii}}-\mathrm{O} 3-\mathrm{Na} 1$ & $108.60(18)$ \\
\hline $\mathrm{Mo}^{\mathrm{xii}}-\mathrm{O} 3-\mathrm{Na} 2^{\mathrm{xiv}}$ & $111.7(2)$ \\
\hline $\mathrm{Na} 1-\mathrm{O} 3-\mathrm{Na} 2^{\mathrm{xiv}}$ & $101.42(16)$ \\
\hline $\mathrm{Mo} 1^{\mathrm{xii}}-\mathrm{O} 3-\mathrm{Na} 2^{\mathrm{xv}}$ & $147.5(2)$ \\
\hline $\mathrm{Na} 1-\mathrm{O} 3-\mathrm{Na} 2^{\mathrm{xv}}$ & $100.80(16)$ \\
\hline $\mathrm{Na} 2^{\mathrm{xiv}}-\mathrm{O} 3-\mathrm{Na} 2^{\mathrm{xv}}$ & $74.53(16)$ \\
\hline $\mathrm{Mo}^{\mathrm{i}}-\mathrm{O} 4-\mathrm{Na} 2^{\mathrm{xv}}$ & $123.6(2)$ \\
\hline 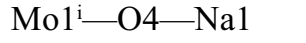 & $117.1(2)$ \\
\hline $\mathrm{Na} 2^{\mathrm{xv}}-\mathrm{O} 4-\mathrm{Na} 1$ & $98.12(17)$ \\
\hline $\mathrm{Mo} 1^{\mathrm{i}}-\mathrm{O} 4-\mathrm{Na} 2^{\mathrm{xiv}}$ & $151.0(2)$ \\
\hline $\mathrm{Na} 2^{\mathrm{xv}}-\mathrm{O} 4-\mathrm{Na} 2^{\mathrm{xiv}}$ & $67.71(15)$ \\
\hline $\mathrm{Na} 1-\mathrm{O} 4-\mathrm{Na} 2^{\mathrm{xiv}}$ & $83.91(14)$ \\
\hline
\end{tabular}
$92.28(15)$
$90.1(2)$
$85.4(2)$
$176.8(2)$
$92.28(15)$
$85.4(2)$
$176.8(2)$
$90.1(2)$
$92.28(15)$
72.09 (19)
$158.89(15)$
90.55 (13)
$90.55(14)$
$158.89(15)$
108.7 (2)
$89.66(15)$
$72.06(14)$
$73.23(14)$
$120.99(15)$
$72.06(14)$
$89.65(15)$

Symmetry codes: (i) $-x+2 / 3,-x+y+1 / 3,-z+5 / 6$; (ii) $-y+4 / 3,-x+2 / 3, z-5 / 6$; (iii) $y-1 / 3, x+1 / 3,-z+5 / 6$; (iv) $y-1 / 3, x+1 / 3,-z+11 / 6$; (v) $-y+1, x-y+1, z$; (vi) $-x+y,-x+1, z$; (vii) $x-y+2 / 3,-y+4 / 3,-z+11 / 6$; (viii) $-x+2 / 3,-x+y+1 / 3,-z+11 / 6$; (ix) $x-y+2 / 3, x+1 / 3,-z+1 / 3$; (x) $-x+y, y, z-1 / 2$; (xi) $y,-x+y,-z+1$; (xii) $y-1 / 3,-x+y+1 / 3,-z+1 / 3$; (xiii) $-y+2 / 3,-x+4 / 3, z+5 / 6$; (xiv) $x-y, x,-z+1$; (xv) $-x+y, y, z+1 / 2$. 